INRA Prod. Anim.. 1990, 3 (5), 347 - 354
Martine NAPOLEONE, T: GILLET $\left.{ }^{*}\right)$

INRA SAD Unité d'Ecodéveloppement Domaine Saint-Paul

84143 Montfavet

$\left(^{*}\right)$ Centre d'Etude et

de Réalisation Pastorale (CERPAM)

Route de la Durance

04100 Manosque

\section{Profil de production du troupeau et système d'élevage caprin de la région Provence Côte d'Azur}

En mettant en relation la courbe de production laitière du troupeau et les pratiques de conduite des exploitants, nous tenterons de comprendre les logiques de fonctionnement d'exploitations caprines laitières de la région Provence - Alpes - Côte d'Azur, a priori très diverses, et de raisonner leur capacité à valoriser l'espace pastoral. Plus que l'élaboration d'une typologie de fonctionnement, le but de ce texte est d'apporter des éléments de réflexion quant à l'intérêt d'aborder des exploitations par l'analyse des pratiques de leurs gestionnaires.

Les espaces pastoraux de la région Provence Alpes Côte d'Azur (PACA) sont largement exploités par des éleveurs caprins spécialisés. Ces élevages présentent une importante diversité de type de troupeau, de mode de conduite, et de productions laitières réalisées.

Il importe de savoir si ces différences sont imputables à des niveaux de maîtrise technique différents, ou à des objectifs particuliers. Ce diagnostic nécessite "la compréhension des projets individuels et la connaissance du fonctionnement de l'exploitation » (Capillon et Manichon 1978). Cependant, les choix individuels ne sont pas toujours facilement perceptibles au regard des informations classiquement

\section{Résumé}

Certains résultats d'élevages caprins, analysés en terme de dynamique, en particulier l'allure de la courbe de production laitière, peuvent être des indicateurs permettant de typer des exploitations en prenant en compte leur fonctionnement. Nous avons ainsi pu mettre en évidence des logiques de fonctionnement différentes dans les élevages caprins laitiers de la région Provence - Alpes - Côte d'Azur. Celles-ci peuvent être dictées par les conditions de milieu (exploitation de montagne) ou peuvent être la conséquence des choix individuels des éleveurs (exploitations de plaine orientées vers la production laitière soutenue ou vers une utilisation importante du milieu pastoral). Ces divers objectifs se traduisent aussi bien au niveau des stratégies de conduite du troupeau (alimentation - production - gestion des lots d'animaux...) qu'au niveau de la gestion économique de l'exploitation. Dans ce contexte, il apparaît nettement que les possibilités d'intégration du milieu pastoral (niveau - durée - période) dans les calendriers d'alimentation de troupeaux caprins laitiers seront d'abord et avant tout liées aux objectifs de chaque éleveur. collectées sur les exploitations (bilans techniques et économiques annuels - caractéristiques struclurelles de l'exploitation). Un ou plusieurs indicateurs qui pourraient être reliés aux choix de conduite refléteraient les objectifs de production de l'éleveur et présenteraient donc un intérêt particulier pour proposer un conseil technique adapté.

Ainsi, en 1986, un groupe de travail INRASAD, CERPAM, EDE de la région PACA a essayé, à partir des données collectées lors de suivis réalisés en exploitations, de :

- dégager un (ou des) indicateur(s) de conduite facilement disponible(s) sur une exploitation. En particulier, le groupe s'est interrogé sur la possibilité de mettre en relation le profil de la courbe de production du troupeau et les différents éléments de conduite de l'exploitation pour aider à identifier les logiques de fonctionnement de chaque système observé.

- raisonner la place et le rôle des parcours et du pâturage dans la conduite de l'alimentation d'élevages caprins producteurs de lait.

\section{1 / Conduite de l'étude}

\section{1 / Les exploitations}

En 1985, onze exploitations caprines de la région P.A.C.A. ont été choisies à partir des critères suivants: répartition géographique permettant de représenter des situations de plaine, de montagne, de littoral et d'arrière pays ; être 
bien connues par les différents services techniques ; ne pas être en phase d'installation ou de modification profonde de l'exploitation ; disposer d'un contrôle laitier sur les animaux traits ; utiliser le pâturage dans leur système d'alimentation.

Dans les exploitations retenues, le troupeau caprin varie de 12 à 80 chèvres. Le niveau de production moyen s'échelonne entre 200 et $1000 \mathrm{~kg}$ de lait par chèvre et par an. Les éleveurs transforment le lait « à la ferme » et commercialisent eux-mêmes leur fromage. Quelques éleveurs vendent leur production en caillé frais.

Excepté dans deux élevages Varois, le troupeau représente une seule entité : tous les animaux sont de même race, orientés vers la production laitière, et reçoivent la même alimentation. La lutte se déroule à l'automne et la mise bas en janvier - février. L'allaitement des chevreaux par leur mère peut varier de 0 à 30 jours. La traite a lieu deux fois par jour, de la mise en traite au tarissement (novembre).

Les deux élevages Varois se composent de 2 et de 3 groupes d'animaux qui diffèrent par leur type génétique, par leur rationnement en chèvrerie et par la conduite des productions (date de mise à la traite après mise bas). L'allaitement dure en moyenne de 1 à 3 mois pour les animaux en production laitière, et de 4 à 6 mois pour les animaux non traits. La traite est effectuée une fois par jour, de la mise en traite au tarissement (novembre ou décembre). Dans ces deux élevages le lot d'animaux constitue l'unité de gestion. Il représente l'unité observée et analysée.

En règle générale, les troupeaux pâturent des prairies ou/et des parcours, et sont complémentés en chèvrerie avec des fourrages et/ou des concentrés. Cependant, la nature du fourrage pâturé et de l'aliment distribué, la part relative de chacun d'eux et le calendrier d'alimentation $\mathrm{du}$ troupeau sont variables d'une exploitation à l'autre.

\section{Le troupeau peut être composé de plusieurs types d'animaux : alpin ou saanen, rove (race locale), commun (popu-} lation de pays).

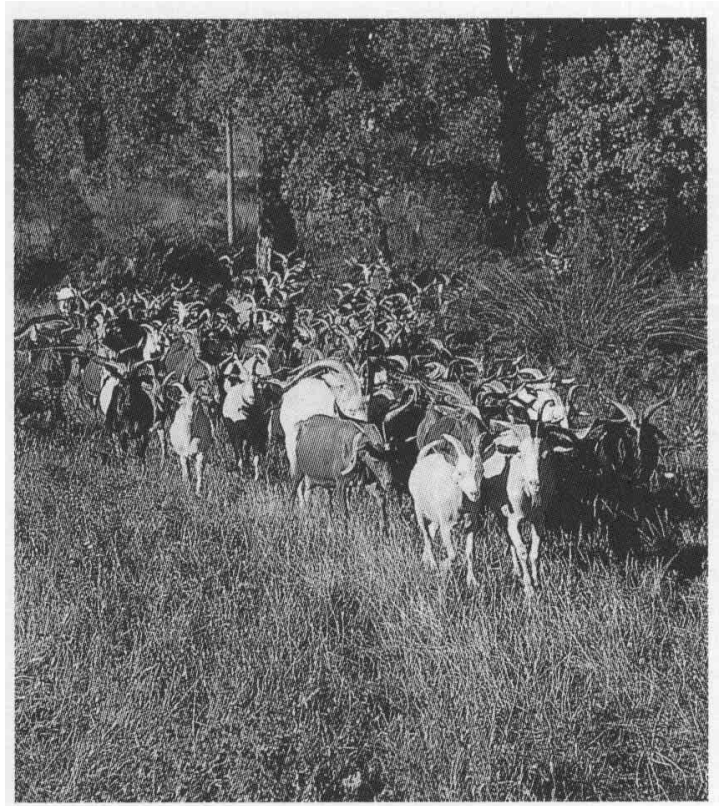

\section{2 / Recueil et analyse des données}

Les données ont été recueillies dans le cadre des suivis techniques et économiques classiques (contrôle laitier, suivi technico-économique "ATEC»), complétés par des enregistrements quotidiens, effectués par l'éleveur, de l'alimentation distribuée en chèvrerie.

Les différents élevages ont été analysés en privilégiant une approche dynamique de certains éléments de leur conduite. Un bilan technique, pour une seule année, n'aurait représenté qu'une image statique ne permettant pas de comprendre une stratégie.

Emettant l'hypothèse que l'éleveur oriente la conduite du troupeau pour atteindre un objectif de production (volume et répartition), ces élevages ont été regroupés à partir de l'évolution mensuelle de la production du troupeau (ou du lot), ramenée à l'animal en production, en comparant de visu les 11 profils étudiés, et en précisant ce classement par une analyse factorielle de correspondance (A.F.C.) (logiciel LEAS). En effet, l'AFC permet de regrouper des lactations sur des profils (Regalgo et Rehben 1979). Les variables actives considérées pour l'analyse factorielle sont les pourcentages mensuels de la production annuelle d'un animal moyen de chaque troupeau. La production mensuelle a été calculée en ramenant au mois la moyenne de production brute entre deux contrôles laitiers consécutifs. Le pourcentage mensuel de la production totale a été obtenu en divisant la production mensuelle estimée par la production totale évaluée par la méthode FLEICHMAN. Pour travailler sur un tableau homogène, seuls les huit premiers mois de lactation ont été retenus. Aucune variable supplémentaire ne fût introduite.

Après avoir ainsi regroupé les lots d'animaux sur des profils de production, nous avons cherché s'il existait à l'intérieur de chaque groupe une cohérence quant :

- à la conduite de l'alimentation distribuée en chèvrerie (évolution mensuelle de l'énergie distribuée). Ces apports ont été estimés à partir des enregistrements quotidiens des quantités moyennes distribuées par individu de chaque lot de conduite homogène. Les valeurs retenues sont de 0,6 UFL/kg MS pour du foin de luzerne ou de prairie de montagne, 0,55 UFL/kg MS pour du foin de pré de plaine, $1 \mathrm{UFL} / \mathrm{kg}$ brut pour l'orge, ou le concentré chèvre laitière.

- à la place accordée à l'alimentation prélevée à l'extérieur (répartition et importance), évaluée à partir de l'évolution de la quantité d'énergie estimée prélevée au pâturage. Cette estimation a été obtenue en calculant, pour chaque mois, ce que l'éleveur ne distribue pas à l'auge : différence entre les apports en chèvrerie et les besoins stricts d'un animal moyen de chaque lot, (hors dépenses liées aux déplacements et aux variations de poids). L'évaluation de ces besoins a été réalisée à partir des recommandations de Morand-Fehr et Sauvant (1988).

- aux grandes « lignes » de la gestion technicoéconomique

Le niveau de réflexion est donc celui de l'unité de gestion: le lot ou le troupeau. Pour 
Figure 1a. Courbes de lactation du troupeau ou du lot des élevages étudiés selon le numéro de contrôle.

11 GROUPES D'ANIMAUX

3 PROFILS DE LACTATION

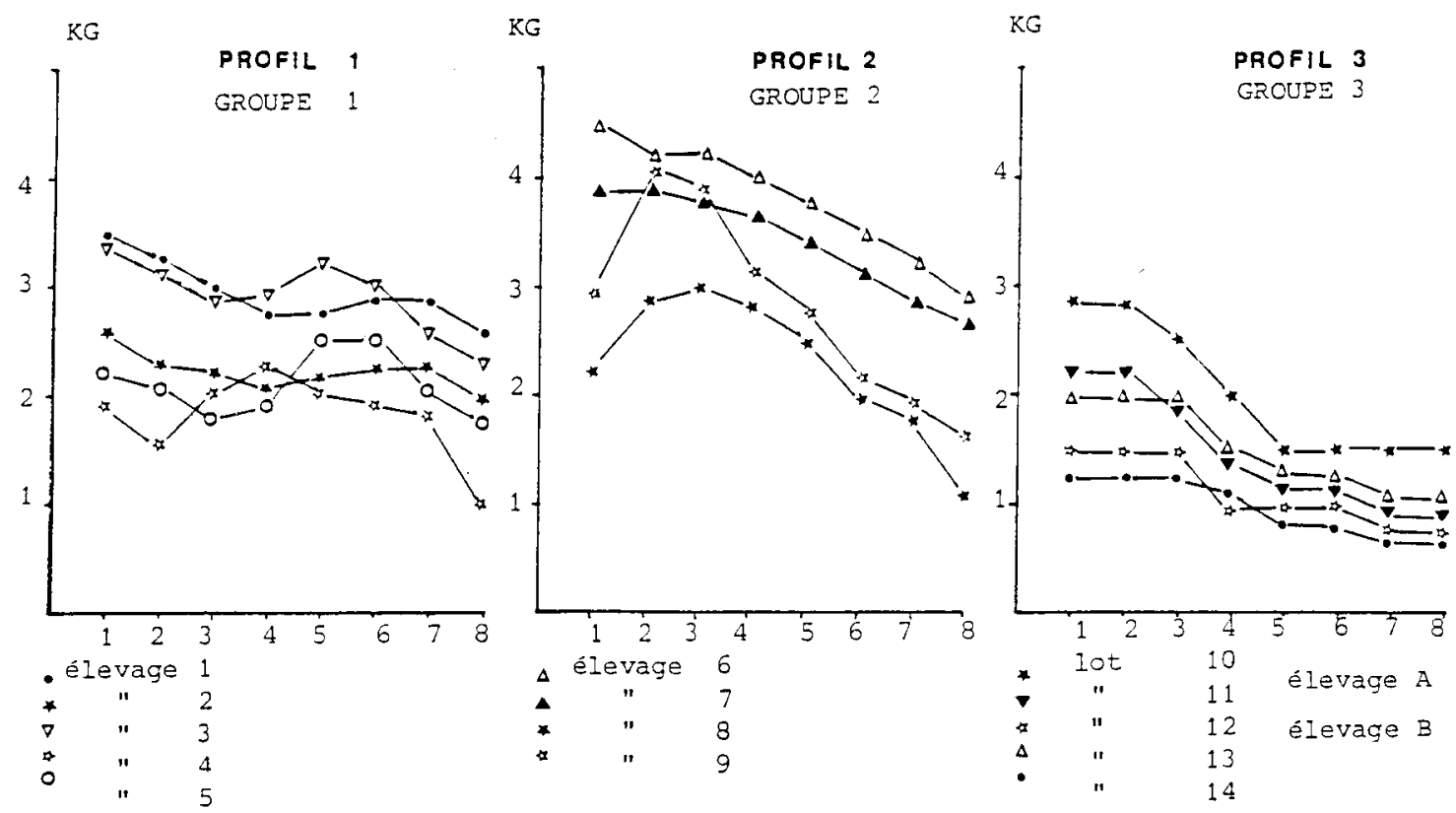

pouvoir comparer les exploitations, toutes les données ont été ramenées à l'individu présent, compte tenu de l'effectif du troupeau.

\section{2 / Résultats}

\section{1 / Profil de production du troupeau}

L'observation visuelle des 14 courbes de production et l'AFC, opposent plusieurs profils à partir des critères suivants :

- différence quantitative de production entre le premier et le huitième mois de production, (l'axe 1 de l'AFC, qui explique $81 \%$ de la variance, oppose ces deux valeurs);

- évolution en milieu de lactation, (forme concave, convexe, ou plane), l'axe 2 de l'AFC, ( $15 \%$ de la variance), oppose les valeurs moyennes aux valeurs extrêmes ;

- évolution de la lactation en phase de démarrage. L'axe 3 , bien que plus délicat à interpréter, fait intervenir notamment l'évolution de la production entre le premier et le troisième mois, ( $2 \%$ de la variance).

Trois groupes de profils ont été retenus (figures $1 \mathrm{a}$ et $1 \mathrm{~b}$ ) :

- Groupe 1: courbe avec chute de production en début de lactation, stabilisation et/ou augmentation de la production vers le quatrième mois et une bonne persistance par la suite ;

- Groupe 2 : production maximum entre un et deux mois de lactation, chute régulière par la suite, persistance mensuelle d'environ $90 \%$;
Figure 1b. Projection des profils de lactation de chaque troupeau sur les plans factoriels 1-2 de l'AFC.

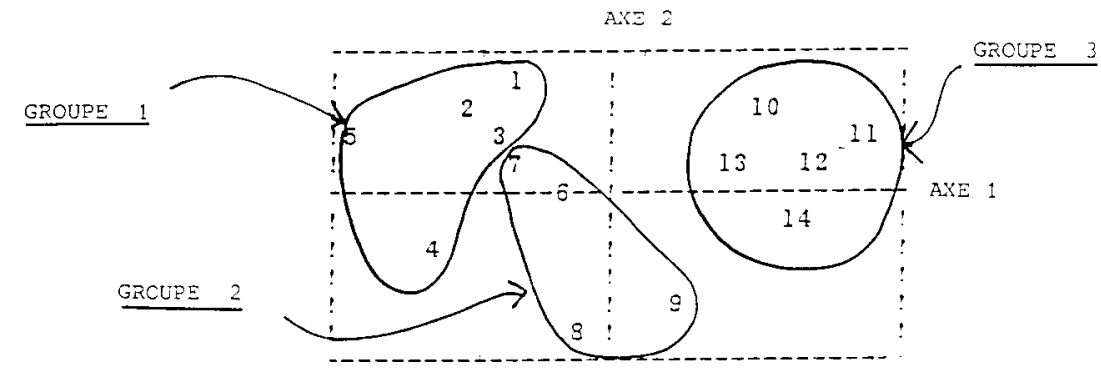

- Groupe 3: courbe sans pic de production marqué, présentant deux plateaux : une forme sensiblement plane du début au milieu de la lactation, puis une chute plus ou moins accentuée en milieu de campagne, et enfin un nouveau plateau jusqu'à la fin de la lactation.

\section{2 / Analyse des modes de conduite de chaque groupe}

\section{a / Conduite de la distribution d'aliment complémentaire en chèvrerie}

Les profils de distribution en chèvrerie, présentés à la figure 2 font apparaître des variations importantes d'un groupe à l'autre.

- Groupe 1: (profil de lactation $\mathrm{n}^{\circ} 1$ ), apports complémentaires en chèvrerie importants en début et en fin de saison. Toute distribution de fourrage sec est suspendue de juin à octobre. L'apport de concentré est régulier, environ 0,6 
Figure 2. Estimation de l'énergie distribuée en chèvrerie, sous forme de foin et de concentré, par groupe d'élevage.

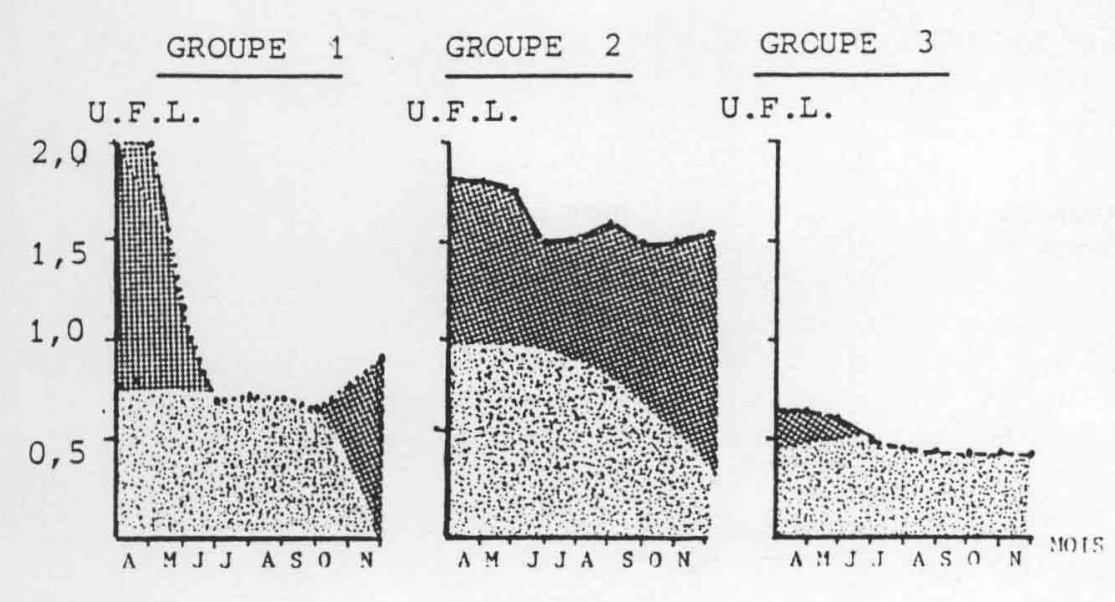

UFL annortées sous forme de foin

UFL apportées sous forme de concentré

à $0,7 \mathrm{~kg} /$ chèvre $/ \mathrm{jour}$, sur toute la période de lactation.

- Groupe 2 : (profil de lactation $n^{\circ} 2$ ), distribution élevée sur toute la période d'enregistrement, équivalente aux besoins stricts d'un animal produisant 2 litres de lait. Le concentré distribué à concurrence de 0,8 à $1 \mathrm{~kg} / \mathrm{animal}$ en début de campagne est progressivement réduit à $0,4 \mathrm{~kg}$ en fin de saison. Un apport de foin est toujours maintenu en chèvrerie, même au printemps, période où l'herbe pâturée est abondante (plus d'un kilo par animal au printemps et en automne, 0,5 à $0,8 \mathrm{~kg}$ par animal en été).

Figure 3a. Evolution mensuelle de l'énergie totale distribuée et de la lactation du troupeau.
- Groupe 3 : (profil de lactation n ${ }^{\circ} 3$ ), l'apport en chèvrerie est régulier durant toute la période de production et se limite pratiquement à un apport d'aliment concentré, (en moyenne 0,4 $\mathrm{kg}$ /chèvre/jour)

\section{b / Relation entre production et complémentation}

L'évolution des performances mensuelles et celle des quantités d'aliments distribuées en chèvrerie aux mêmes périodes, divergent d'un groupe à l'autre. La figure 3a précise la relation existante entre ces deux variables, relation qui peut exprimer les décisions de conduite des éleveurs pour réaliser une production donnée.

Les exploitants du groupe 1 réalisent « l'apport total ", (couverture d'environ la totalité des besoins d'un animal moyen) jusqu'en mai. De juin à octobre ils ne distribuent qu'environ l'équivalent des besoins de lactation. A partir de novembre, la part du besoin couverte par la ration en bergerie tend à nouveau vers la totalité.

Les éleveurs du groupe 2 distribuent toujours plus que les besoins de lactation, et maintiennent un niveau d'apport régulier. Avec l'avancement de la lactation une part de plus en plus importante du besoin est couverte par le distribué. Au tarissement, l'éleveur apporte plus que la totalité des besoins. Ces exploitants paraissent donc ainsi préparer la lactation suivante par une alimentation libérale des animaux. Fn outre, (figure 2), la proportion de concentré dans la ration est progressivement réduite du début à la fin de la campagne ; l'apport d'énergie par la complémentation sous forme d'aliments concentrés tend à chuter.

Les éleveurs du groupe 3, couvrent uniquement les besoins de lactation de leurs animaux,

Foin et concentré distribue

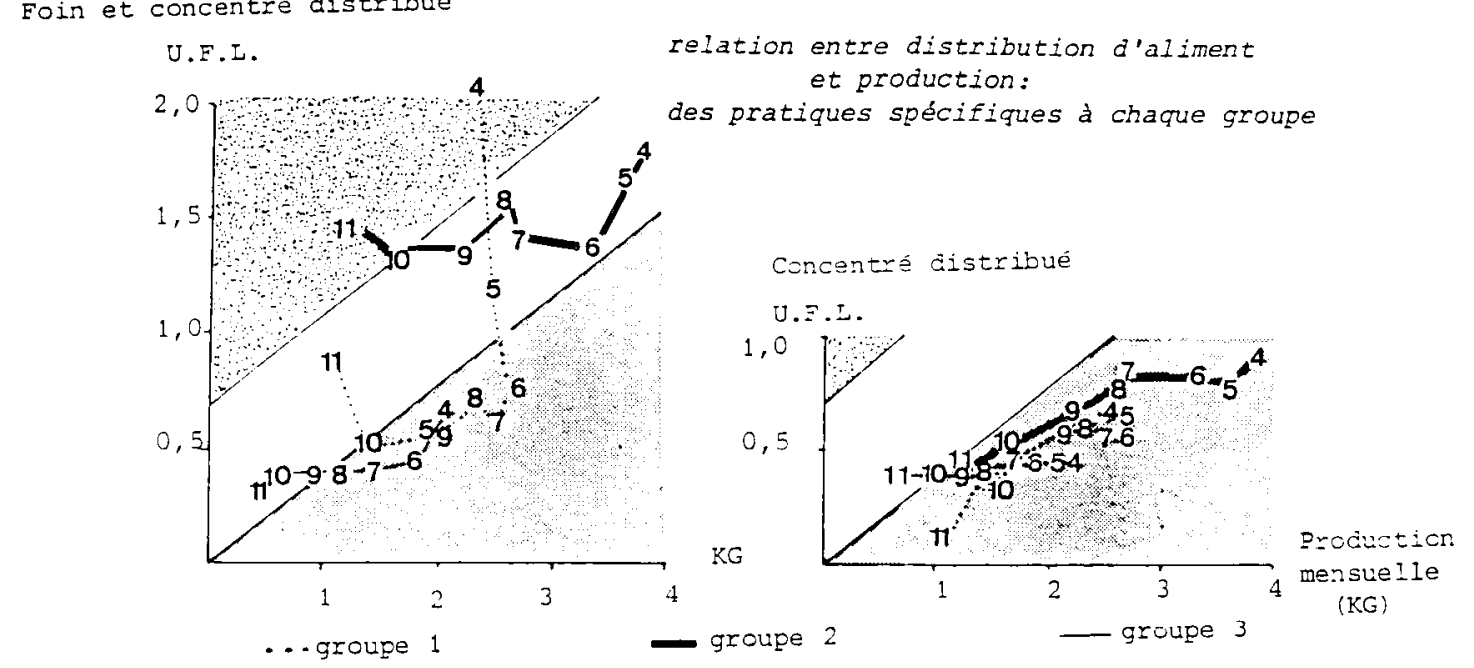

distribué inférieur aux besoins de production

istribú compris poduction et les besoins dentretien

distribur besoins de production et d'entretien

distribué supérieur aux besoins de production et d'entretien,

Figure $3 \mathrm{~b}$ Evolution mensuelle de l'énergie distribuée sous forme de concentré et de la lactation du troupeau. 
quels que soient la saison ou le stade physiologique, et cessent tout apport au tarissement.

Si la quantité totale d'UFL distribuée en chèvrerie relève d'une logique spécifique à chaque groupe d'exploitation, la distribution d'aliment concentré semble être raisonnée sur les mêmes bases et dans le même but (figure $3 \mathrm{~b}$ ) : tout semble se passer comme si, les exploitants accompagnent directement la production laitière à partir d'aliment distribué sous forme de concentré.

\section{c / Place et rôle du pâturage}

Puisque les exploitants conduisent différemment l'alimentation en chèvrerie, le pâturage, qu'ils utilisent tous, n'occupe pas la même place dans le rationnement des animaux. Cette place peut être estimée, en évaluant l'énergie prélevée sous forme de fourrage sur pied, à partir du calcul de ce que l'éleveur ne distribue pas en faisant la différence entre les besoins (hors dépenses liées aux déplacements et aux variations de poids), et les apports (figure 4).

Pour les exploitations des groupes 1 et 2 un prélèvement important n'est observé que périodiquement lorsque les pâturages sont de bonne valeur fourragère. Dans les élevages du groupe 3, l'intensité de ce prélèvement est élevée, quelle que soit la saison, puisque celui-ci doit toujours couvrir au moins les besoins d'entretien.

Il semblerait donc que pour certains exploitants, la décision d’intégrer le pâturage puisse être liée à l'évolution qualitative de celui-ci, (groupe 1 et 2). Par contre, pour les éleveurs du groupe 3 , la ressource pâturée représente la base de l'alimentation et son intégration dans le calendrier d'alimentation présente peu de variation.

On peut, d'autre part, tenter de raisonner « le niveau d'exigence qualitative » de l'éleveur par rapport au fourrage sur pied, à partir d'une démarche théorique appliquée, tenant compte de l'animal et du distribué en chèvrerie, seules informations disponibles, (la composition des prélèvements et leur valeur alimentaire ne sont
Figure 4. Estimation de l'évolution des prélèvements énergétiques au pâturage.

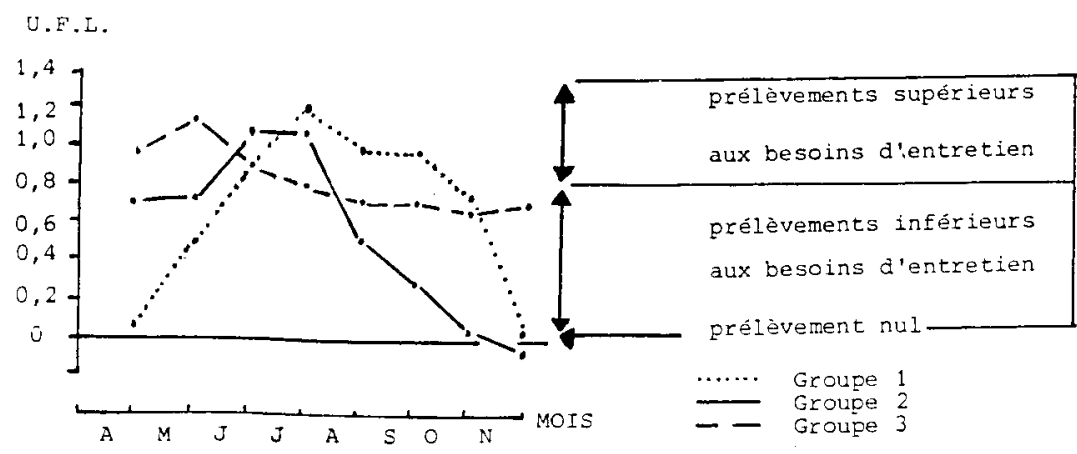

pas connues]. Utilisons pour cela la notion de matière sèche volontairement ingérée par un animal, tentons de l'appliquer à l'alimentation mensuelle du troupeau, et analysons le résultat en terme d'indicateur.

Sauvant et Morand-Fehr (1977) ont mis au point, en situation contrôlée, des abaques qui permettent d'estimer la quantité de fourrage grossier susceptible d'être ingérée, à partir de la production de lait et de l'apport d'aliment concentré. A partir de ces abaques, ont été évaluées les quantités totales de fourrages grossiers théoriquement ingérées par un animal moyen de chaque troupeau, et par déduction de la quantité de foin consommée en chèvrerie, les quantités de fourrage sur pied ingérées. Connaissant la quantité d'énergie devant être prélevée hors chèvrerie pour assurer la couverture des besoins énergétiques de cet animal moyen, on peut alors estimer la concentration énergétique de ce prélèvement, (C.E. exprimée en UFL/kg de MS).

S'il est hors de question d'utiliser les résultats obtenus pour raisonner sensu stricto un rationnement, puisque leur précision est inconnue, il est intéressant de rapprocher leurs évolutions de celles des quantités estimées prélevées au pâturage, et d'analyser la relation existante entre "attente quantitative et exigence qualitative».

Figure 5. Evolution de la quantité d'UFL estimée à prélever au pâturage en fonction de l'estimation de la concentration énergétique de ce prélèvement.

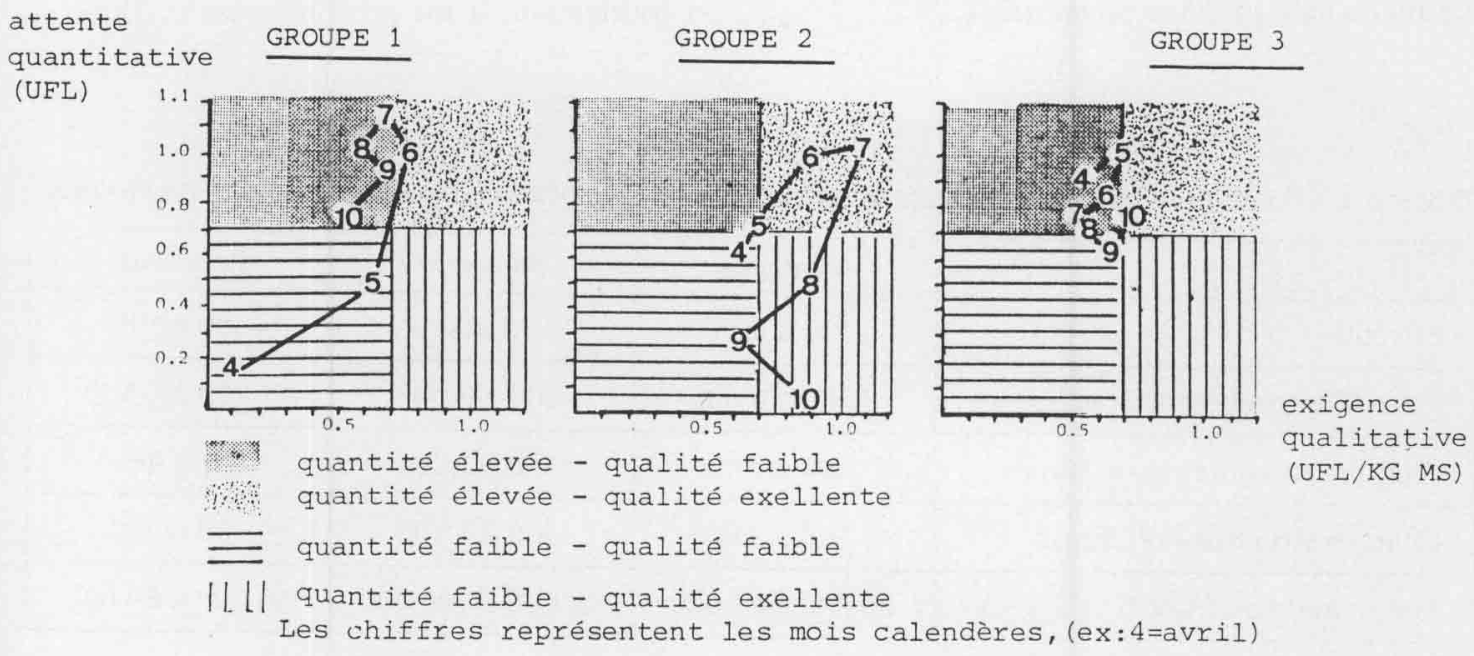

quantité élevée - qualité faible

1\% quantité élevée - qualité exellente

exigence qualitative (UFL/KG MS)

三 quantité faible - qualité faible

II. U quantité faible - qualité exellente

Les chiffres représentent les mois calendères, (ex:4=avril) 
Si l'importance quantitative du fourrage sur pied dans la ration varie entre les groupes d'exploitation, « l'exigence qualitative » de l'éleveur qui conduit son troupeau au pâturage diffère elle aussi (figure 5).

Pour les élevages du groupe 1 ou pour ceux du groupe 3 , la qualité du prélèvement reste moyenne durant la période où sa participation quantitative dans la ration est importante. Une complémentation sous forme de concentré, en période de pâturage, limite de fait la concentration énergétique que le prélèvement devrait présenter.

Par contre, pour les élevages du groupe 2, le pâturé doit être d'excellente qualité durant les 2 à 4 mois où sa participation à la ration n'est pas négligeable (plus de 0,6 à 0,7 UFL).

Donc les différentes conceptions de conduite impliquent que les modes de gestion des ressources (type de ressource utilisée, période d'utilisation et mode d'utilisation) soient différents.

\section{d / Profil de lactation du troupeau et orientations technico-économiques.}

A l'analyse du tableau 1 on note que les exploitations des groupes 1 et 2, dont les troupeaux présentent des productions moyennes relativement élevées, se sont engagées dans un processus d'intensification. Ce choix implique que la gestion des facteurs de production (capital-troupeau et travail principalement) soit orientée vers une rentabilisation maximum (Tirel 1987): les charges alimentaires et de structure sont élevées, mais le produit brut dégagé par chaque chèvre est important et l'effectif du troupeau réduit.

Dans les exploitations pastorales (groupe 3) par contre, il n'apparaît pas d'objectif de " maximisation" des facteurs de production: le produit brut par chèvre, ainsi que les charges alimentaires et de structures par chèvre sont faibles. La production de l'élevage est réalisée à partir d'une combinaison des productions élaborées par des groupes d'individus aux potentialités différentes (Napoléone et Hubert 1987). Le produit de l'exploitation est alors obtenu par un effectif élevé d'individus peu exigeants, nécessitant peu de charges. Avec cette organisation, l'éleveur module la production laitière du troupeau, malgré une faible maîtrise de la ration de base prélevée au pâturage.
Le revenu agricole semble dans certains cas directement lié à la productivité des individus, mais dans d'autres à celle du troupeau (Weber 1986) : si la marge nette semble relativement équivalente entre ces exploitations, son mode d'élaboration diffère d'un groupe à l'autre.

\section{3 / Discussion}

\section{1 / Plusieurs systèmes d'élevage}

Il existe donc pour les trois groupes identifiés à partir du profil moyen de production du troupeau une relation entre le type de produit obtenu, la gestion des « outils techniques »utilisés par l'éleveur, ainsi que les résultats technico-économiques réalisés au niveau de l'exploitation.

- Groupe 1 : ce sont des exploitations de montagne (800 à 1000 mètres d'altitude), où l'hiver est rude, (une période de chèvrerie est obligatoire). Dans ce contexte, des animaux présentant un bon potentiel de production permettent une bonne valorisation de cette période d'affouragement en sec. Pour un même volume de production, l'effectif du troupeau est plus réduit, et le besoin total sera composé en majeure partie des dépenses liées au niveau de production. L'affouragement en sec sera donc plus utilisé par le troupeau pour assurer sa production que pour son entretien. La production laitière moyenne du troupeau est élevée (500 à $700 \mathrm{~kg} /$ chèvre/an), et les quantités moyennes distribuées en chèvrerie importantes $[0,65$ à 1,05 UFL/jour), mais principalement distribuées hors de la période de pleine productivité des prairies de montagne. Avec une mise bas en janvier-février, les besoins maxima des animaux correspondent à la période où les stocks hivernaux s'épuisent, et où toute pâture est impossible. La lactation, limitée par le niveau de l'apport en chèvrerie, démarre mal, mais dès la mise à l'herbe elle retrouve une évolution normale (figure 1). Il est intéressant de constater que la lactation de ces animaux au potentiel de production élevé (niveau actuel 500 à 700 $\mathrm{kg}$ ), augmente à partir du cinquième mois malgré un niveau alimentaire bas qui nuit au démarrage de la lactation. Le choix d'une mise bas précoce limite donc le volume de production du troupeau. Mais avec le tourisme hivernal, la production peut être écoulée facilement et localement, à un prix intéressant. Donc, la

Tableau 1. Effectif du troupeau et résultats économiques de l'exploitation dans les trois groupes étudiés.

\begin{tabular}{|l|c|c|c|}
\hline & Montagne & Soutenue & Pastoraux \\
\hline Effectif & 16 à 49 & 13 à 41 & 80 à 100 \\
\hline Produit brut/chèvre/lot en francs & 3000 à 6000 & 4000 à 7000 & 1400 à 2300 \\
\hline Charges alimentaires en francs & 400 à 1000 & 700 à 1200 & 200 à 400 \\
\hline Charges structures en francs & 900 à 2600 & 400 à 1100 & 80 à 300 \\
\hline Marge nette en francs & 20000 à 41000 & 42000 à 100000 & 50000 à 80000 \\
\hline
\end{tabular}


réalisation de la production est conditionnée ici par le choix du calage de la date de mise bas, qui résulte d'un compromis entre des critères techniques, et des considérations d'ordre économique.

- Groupe 2 : Ce sont des exploitations de plaine, que nous pourrions appeler à «production soutenue». Suivant le schéma développé dans le Centre Ouest, leur stratégie est fondée sur l'expression maximum du potentiel des animaux pour rentabiliser au mieux le capital et le travail. C'est le modèle laitier, pouvant aller du hors sol à un système mixte utilisant des aliments conservés et du pâturage sur les surfaces fourragères. Le troupeau est composé d'individus sélectionnés, de génotypes alpins ou saanens. La production laitière moyenne est élevée $(500$ à $1000 \mathrm{~kg})$. L'alimentation distribuée, importante toute l'année (plus de 1,4 UFL/ femelle/jour en moyenne), est calculée pour soutenir un bon niveau de production (figures 2 et 3a). Ainsi, en fin de lactation, par le maintien d'une complémentation élevée, les éleveurs préparent la lactation à venir.

Bien que le troupeau puisse être conduit au pâturage une grande partie de l'année, l'éleveur n'intégrera la participation de celui-ci dans le rationnement de ses animaux que lorsque, sa valeur fourragère est supérieure ou égale à celle d'un foin de bonne qualité (plus de 0,7 UFL $/ \mathrm{kg}$ MS) (figure 5). L'objectif prioritaire n'est pas en effet de réduire le coût de l'alimentation distribuée mais d'obtenir un bon niveat de production laitière puisque la valeur ajoutée dégagée par chaque animal est élevée.

- Groupe 3 : ce sont aussi des exploitations de plaine, créées dans la mouvance des années 70 (Gauthier 1983), mais dont les facteurs de production sont organisés de manière à utiliser au maximum le milieu pastoral (figure 4). Cette hypothèse de base implique que le modèle laitier évoqué précédemment ne peut être appliqué. Les possibilités de pilotage de la production individuelle par l'alimentation distribuée sont limitées par des facteurs liées au système (qualité et quantité du fourrage pâturé, importance des déplacements). L'éleveur ne peut donc pas miser sur la productivité des individus car il ne peut contrôler la ration de base que devrait fournir le parcours (figure 5). L'éleveur gère ici la production du troupeau à partir de l'effectif et d'une organisation souple des productions (durée de l'allaitement) qui permet de compenser les variations du disponible. Si, à l'intérieur d'un même troupeau, le niveau de complémentation est raisonné pour chaque génotype, la stratégie de distribution est la même quel que soit le niveau de production des animaux. L'éleveur, par le biais de l'aliment distribué, « accompagne » la lactation, sans utiliser cet outil pour accroître la production (figure 3a). Au tarissement toute complémentation est arrêtée.

Il convient de remarquer que le distribué en chèvrerie n'est pas utilisé de la même façon par tous les éleveurs. En élevage de type montagne ou de production soutenue, l'apport en chèvrerie est modulé par rapport aux besoins, compte tenu du potentiel de prélèvement du fourrage sur pied. On se rapproche là du type de raison-

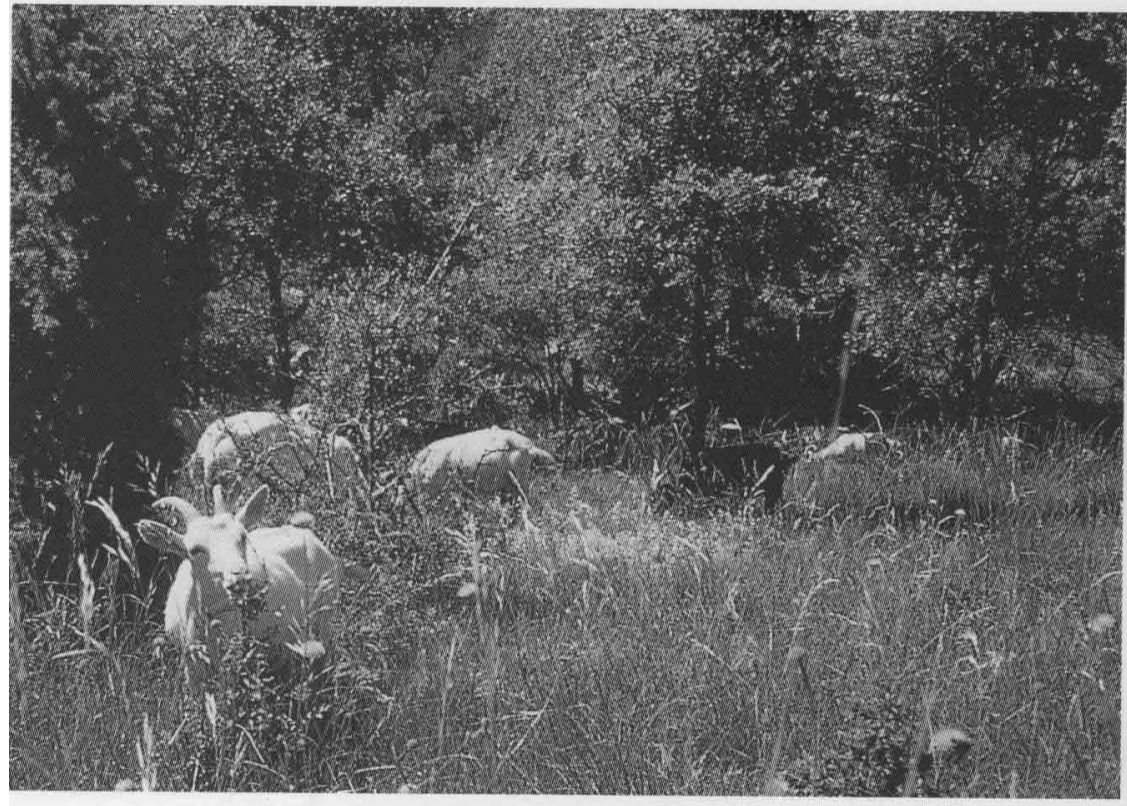

nement employé pour calculer une complémentation. Par contre en élevage pastoral, « le potentiel de prélèvement » intervient dans le choix du matériel génétique (proportion d'animaux sélectionnés dans le troupeau), ainsi que dans l'objectif de production individuelle. Le distribué (essentiellement du concentré), représente alors un appoint ajusté exclusivement sur la production.

\section{2 / A chaque système son conseil...}

\section{... Augmenter la productivité ...des individus, du troupeau, du travail ?}

Il faut donc distinguer production et système productif. Dans le modèle laitier ce système est représenté par une conduite d'individus performants gérés au mieux. Dans le schéma pastoral, l'existence même du troupeau n'est fondée que sur une combinaison des aptitudes des différents groupes du troupeau dont les proportions sont susceptibles d'être réajustées fréquemment en fonction d'un certain nombre de contraintes. La valorisation des actions entreprises semble donc dépendante de la production des individus dans les schémas laitiers, ou de celle de l'ensemble des groupes dans le schéma pastoral.

Ces différences fondamentales impliquent que dans chaque type d'exploitation, l'amélioration de la productivité du travail ne reposera pas sur les mêmes éléments.

En schéma laitier cette productivité est étroitement liée à celle des individus et à une valorisation maximum des intrants mis en jeu. En schéma pastoral, les possibilités d'augmenter le produit brut par animal sont limitées par des facteurs liés au système. La productivité du travail est alors directement dépendante de l'effectif qui permet de s'ajuster sur des économies d'échelle. Le développement de ces exploitations ne pourra donc pas s'appuyer entièrement sur les bases du modèle productiviste courant, ou alors, ce choix impliquerait une remise en cause complète de leur organisation.
Les animaux prélèvent leur ration en pâturant des espaces diversifiés.
Selon le système mis en place la production du troupeau repose sur la productivité individuelle ou sur la souplesse des individus et l'effectif du troupeau. 


\section{... Valoriser l'espace pastoral par des troupeaux caprins laitiers.}

Les différences entre ces trois logiques montrent que le pâturage ne représente pas le même enjeu pour tous les élevages caprins.

En montagne, le système d'élevage est orienté de manière à valoriser l'affouragement en sec. Les prairies ne sont donc intégrées de façon conséquente que lorsqu'elles sont pleinement productives. La valeur fourragère de celles-ci permet aux animaux de prélever une ration de base de bonne qualité. L'utilisation de cette ressource est plus liée à des problèmes d'organisation du territoire (éloignement des parcelles par rapport au lieu de traite, à l'abri, au point d'eau...) que d'amélioration de la qualité du pâturage.

En plaine, schéma laitier, le pâturage remplace un bon foin pendant la période de production des espèces herbacées (figure 4). Son intégration dans le système fourrager des animaux est limitée au printemps et à l'été. Sa valeur fourragère doit être élevée pour garantir une bonne adéquation ressources-besoins (figure 5). Dans ces conditions, l'utilisation de parcours pauvres ou de certains espaces en période de repos végétatif, est envisagé en association avec des ressources fourragères d'excellente qualité afin que la valeur énergétique de la ration soit assez élevée pour assurer les performances de production souhaitées.

Dans les exploitations pastorales, le fourrage sur pied constitue la base du système d'alimentation. Le faible niveau de production des animaux permet une plus grande souplesse dans la conduite de leur alimentation et le mode d'organisation des productions. Des fourrages de qualité moyenne peuvent ainsi être utilisés quelle que soit la saison (figure 5). Le niveau de production étant assez faible, l'éleveur pourra corriger la ration de base prélevée sur les parcours à partir d'un aliment concentré.

\section{Conclusion}

L'approche de ces exploitations par une étude de l'évolution d'un des principaux repères de l'éleveur, à savoir la production de son troupeau facilite la compréhension des objectifs de production. La mise en relation de cet indicateur avec les pratiques de conduite permet d'apprécier la cohérence de chaque système et de raisonner des voies de développement adaptées.

En particulier, en fonction de son objectif de production, chaque exploitant tolère un niveau de contrainte en intégrant plus ou moins l'utilisation d'espaces pastoraux dans le système d'alimentation de son troupeau. Les voies d'amélioration de la production laitière soit de l'individu soit du troupeau devront donc tenir compte de ces contraintes et inversement, les possibilités de valoriser des espaces pastoraux dépendront en partie du mode d'organisation du processus de production.

\section{Remerciements}

Nous remercions D. Baron (EDE 04), H. Coursange (EDE 05), P. Gautier (EDE 83), P. Berthon (EDE 84) pour leur contribution à cette étude.

\section{Références bibliographiques}

CAPILLON A., MANICHON H., 1978. La typologie des exploitations agricoles: un outil pour le conseil technique. In "Exigences nouvelles pour l'agriculture : les systèmes de culture pourront-ils s'adapter " Ed.ADEPRINA INA-PG 16, rue Claude Bernard 75005 Paris. 450-462.

Document de travail INRA-SAD - CERPAM - EDF (04 - 05 - $83-84), 1986$. « Suivi des exploitations d'élevage caprin - résultat de la campagne 1985 ». (non publié).

GAUTIER L.A., 1983. «L'élevage caprin dans le Sud Varois. Analyse de système de production ». ENSAM Montpellier. 129 p.

MORAND-FEHR P., SAUVANT D., 1988. "Alimentation des Caprins ". in Alimentation des bovins, ovins et caprins. Ed. R. Jarrige, INRA Pub., 281-304.

NAPOLEONE M., HUBERT B., 1987. "Caractériser et évaluer des systèmes de production caprins, fortement utilisateurs de parcours : un exemple dans le Sud-Est de la France ». Symposium FAO, 23-25 septembre 1987 Portugal.

REGALGO D., REHBEN E., 1979. "Contribution à l'étude des courbes de lactation en vue de la prévision». Mémoire de fin d'étude. INA-PG - ITOVIC. 86 p. + annexes.

SAUVANT D., MORAND-FEHR P., 1977. «Méthodes de rationnement de la chèvre laitière ». Murcia - Granada Malaga. 3-7 octobre 1977.

TIREL J.C., 1987. «Intensification hier? Extensification demain?" INRA - Direction des Politiques Régionales.

WEBFR L., 1986. «Conditions du développement des élevages caprins en zones sèches: approche technico-économique ». Doc. INRA-SAD - Ecodéveloppement ENSSAA Dijon. 46 p. + annexes.

\section{Summary}

Caprine production profiles and breeding systems in the Provence and Côte d'Azur regions.

The analysis of breeding results, in particular the milk production curve, could be regarded as an operation indicator to identify different farm types. Thus, we have identified several functioning systems in dairy goat farms in Provence and the Côte d'Azur. Some are related to the environment (mountains farms) others to the farmer's aims (dairy intensification in plains or large amount of usage of rough grazing). These various targets involve differences in herd management (feeding, pro- duction, allotment,...) and in economic management. Consequently, the level of integration of rough grazing into the feeding systems is closely dependant on the aims of each farmer and the farm's technical contraints they have to take into account. Our aim is to provide a tool in order to easily understand this integration.

NAPOLEONE Martine, GILLET G., 1990. Profil de production du troupeau et système d'élevage caprin de la région Provence Côte d'Azur. INRA, Prod. Anim., $3(5), 347$ - 354 